\title{
Effect of intermonolayer coupling on the phase behavior of lipid bilayers
}

\author{
Zhang, Zhengping; Zuckermann, Martin J.; Mouritsen, Ole G.
}

Published in:

Physical Review A

Link to article, DOI:

10.1103/PhysRevA.46.6707

Publication date:

1992

Document Version

Publisher's PDF, also known as Version of record

Link back to DTU Orbit

Citation $(A P A)$ :

Zhang, Z., Zuckermann, M. J., \& Mouritsen, O. G. (1992). Effect of intermonolayer coupling on the phase behavior of lipid bilayers. Physical Review A, 46(10), 6707-6713. https://doi.org/10.1103/PhysRevA.46.6707

\section{General rights}

Copyright and moral rights for the publications made accessible in the public portal are retained by the authors and/or other copyright owners and it is a condition of accessing publications that users recognise and abide by the legal requirements associated with these rights.

- Users may download and print one copy of any publication from the public portal for the purpose of private study or research.

- You may not further distribute the material or use it for any profit-making activity or commercial gain

- You may freely distribute the URL identifying the publication in the public portal 


\title{
Effect of intermonolayer coupling on the phase behavior of lipid bilayers
}

\author{
Zhengping Zhang and Martin J. Zuckermann \\ Department of Physics, Centre for the Physics of Materials, Rutherford Building, McGill University, 3600 University Street, \\ Montréal, Québec, Canada H3A 2T8 \\ Ole G. Mouritsen \\ Canadian Institute of Advanced Research and Department of Physical Chemistry, \\ The Technical University of Denmark, Building 206, DK-2800 Lyngby, Denmark
}

(Received 24 March 1992; revised manuscript received 4 June 1992)

\begin{abstract}
A statistical-mechanical lattice model is proposed to describe the acyl-chain main phase transition in a hydrated lipid bilayer. The model is built on a two-dimensional multistate lattice model to describe the intramonolayer interactions within the two separate lipid monolayers of the bilayer. The coupling between the two monolayers is modeled both indirectly by hydrophobic acyl-chain mismatch interactions that ensure compatibility between the two monolayers, and by a direct intermonolayer attractive dispersion force. The nature of the phase transition is studied by computer-simulation methods involving standard Monte Carlo simulation, as well as the extrapolation method of Ferrenberg and Swendsen [Phys. Rev. Lett. 61, 2635 (1988)] and the Lee-Kosterlitz technique [Phys. Rev. Lett. 65, 137 (1990); Phys. Rev. B 43, 3265 (1991)]. It is found that the absence of a phase transition in a set of uncoupled monolayers is restored by a weak intermonolayer interaction. The bilayer properties in the transition region are described with particular emphasis on the lateral density fluctuations and the resulting dynamic bilayer heterogeneity. The theoretical results are discussed in relation to experimental data.
\end{abstract}

Pacs Number(s): 64.60.Cn, 87.22.Bt

\section{INTRODUCTION}

Lipid molecules are basic components of biological membranes [1]. When these molecules are dissolved in water at sufficiently high concentrations and the mixture is then vortexed, the lipid molecules self-organize to form soft multilamellar structures in which each lamellar sheet is a hydrated lipid bilayer. These systems are known as liposomes or multilamellar vesicles. If these vesicles are subjected to further treatment, for example by irradiation with ultrasound, small and eventually large unilamellar vesicles are formed. Large unilamellar vesicles are regarded as models for biological membranes and can, for example, be reconstituted with membrane-bound proteins, thereby allowing a detailed study of the structure and function of such proteins. Pure lipid bilayers exhibit a considerable degree of polymorphism and their phase behavior has been subject to intensive research activity in recent years, which has led to an understanding of the material properties of lipid membranes in terms of fundamental molecular interactions between the membrane components [2]. In this paper we concentrate on a theoretical description of the main phase transition [3] in pure fully hydrated bilayers composed of saturated phospholipids which are the most common lipid molecules used in experimental studies of model membranes [1].

The main phase transition of lipid bilayers is a structural phase transition in which the bilayer passes from a gel or quasi-two-dimensional solid phase to a liquidcrystalline or quasi-two-dimensional fluid phase [3]. In the gel phase the acyl chains of the lipid molecules are rigid and form a crystalline lattice, whereas the chains become comformationally disordered in the liquidcrystalline phase which is characterized by fast lateral diffusion of the lipid molecules. It is found experimentally that the conformational order of the acyl chains changes abruptly at the transition temperature $T_{m}$, which is signaled by a sharp peak in the thermal response functions, such as the specific heat.

The structure and dynamics of lipid bilayers have been studied using a number of different microscopic interaction models. Considerable insight into details of acyl-chain order and dynamics has been obtained away from the transition region using three-dimensional models with model potentials characterized by a high degree of realism [4]. However, due to the complexity of the interactions it is only feasible to study a small number $\left(\sim 10^{2}\right)$ of molecules with these very realistic models. Since the main transition is associated with strong fluctuations this limits the information obtained to a region away from the transition. It is therefore necessary to introduce simplifications in the models in order to permit a study of large assemblies of molecules comprising at least $10^{3}-10^{5}$ molecules. Reduction to two-dimensional models is an obvious first simplification [5]. In particular, two-dimensional multistate statistical-mechanical lattice models have been very useful in describing the characteristics of the main phase transition $[3,5]$. In these models the states represent the conformations of the acyl chains. The models are usually constructed under the assumption that (i) the main phase transition is entropically driven by the change in acyl-chain order and (ii) the two 
monolayers of the bilayer are essentially independent and noninteracting. The next level of simplification would involve taking into account the interactions between the two monolayers of the lipid bilayer thereby moving into the third dimension. This is the level we are going to approach in the present work.

The lattice model used in the present paper is built on a ten-state conformational lattice model due to Pink [6, 7]. In the Pink model, a single monolayer of the bilayer is represented by a triangular lattice in which each site is occupied by an acyl chain. The model, which is described in Sec. II, has previously been examined in detail both in the mean-field approximation and by extensive Monte Carlo simulations [3,5]. The mean-field calculations were used to obtain values for the model parameters by fitting to thermodynamic data for saturated lecithins [diacyl lipids with phosphatidylcholine (PC) polar heads]. Using these fitted parameters the mean-field solution to the model then predicted sharp first-order phase transitions for saturated lecithin bilayers with acylchain lengths ranging from 12 to 22 carbon atoms. The numerical simulations for the Pink model were performed using these fitted parameters for acyl chains with 14, 16, and 18 carbon atoms [8]. The simulation results showed that the transition appears in the model as continuous but abrupt with a sharp peak in the specific heat. The monolayer was found to be spatially and dynamically heterogeneous in the neighborhood of the transition. Furthermore, Ipsen, Jørgensen, and Mouritsen [8] showed that the lateral density fluctuations could be characterized by a finite length scale and behaved in a pseudocritical manner characteristic of a weak first-order phase transition near a critical point. They also found that the specific heat sharpened with increasing chain length. Recently, Corvera, Laradji, and Zuckermann [9] used the finite-size-scaling method of Lee and Kosterlitz $[10,11]$ to show that a phase transition in the ten-state Pink model is in fact absent for lipid chains with up to 18 carbon atoms and that the dynamic heterogeneity of the system is due to short-range-order effects close to but beyond a critical end point. In an attempt to determine the effects on the phase behavior of interactions left out in the simple ten-state Pink model, Zhang et al. [12] recently extended the Pink model for a single monolayer by including hydrophobic intralayer mismatch interactions $[13,14]$ between the acyl-chain conformational states of the Pink model. These mismatch interactions mimick in an indirect fashion the constraints placed on each monolayer in forming a bilayer. Zhang et al. [12] found that even a rather weak mismatch interaction is sufficient to take the system below the critical end point and to induce a first-order phase transition.

The model and work reported in the present paper are extensions of the work on the model with intralayer mismatch interactions [12] in an attempt to include the forces between the two monolayers of the bilayer. In contrast to our earlier work [12], in which only a finite-size scaling analysis was presented to reveal the nature of the transition, in the present paper we present the results of a finite-size scaling analysis, together with the physical properties of the system for particular values of the model parameters. We concentrate on the case where these parameters lead to a weak first-order phase transition in the thermodynamic limit, as suggested by recent experiments $[15,16]$, and examine the heterogeneous nature of the bilayer in the neighborhood of this transition. We then compare these results with those obtained for a single monolayer which in our model has no phase transition in the thermodynamic limit but is close to a critical point.

Models describing the complete bilayer as a system of interacting monolayers are useful since they can be extended to include and distinguish between peripheral and integral membrane-bound proteins. Furthermore, they allow us to model monomer-dimer dissociations for certain integral proteins. The first interacting lattice model of a complete bilayer is due to Georgallas et al. [17] who used the two-state lattice model due to Doniach $[7,18]$ for each monolayer and then combined the monolayers by making each lattice site of one monolayer an interstitial site of the other monolayer. Weak nearest-neighbor interactions on the composite lattice represent intermonolayer interactions, whereas next-nearest-neighbor interactions represent the usual intramonolayer interactions between acyl chains. It was shown by both analytical and simulation methods [17] that even a weak intermonolayer interaction is sufficient to change the critical point dramatically. Georgallas et al. [17] used the model to explain the absence of a pressure-induced transition in dipalmitoyl phosphatidylcholine (DPPC) monolayers at $41^{\circ} \mathrm{C}$ in contrast to the existence of the main phase transition of DPPC bilayers which takes place at this temperature for the corresponding value of the internal pressure.

In the model proposed in the present paper, each monolayer of the lipid bilayer is described by a tenstate Pink model and the bilayer is constructed by directly matching lattice sites in each monolayer. An interlayer mismatch interaction between different conformational chain states is now imposed across the bilayer together with a single-site van der Waals interaction between chains belonging to different monolayers. The model is presented in Sec. II. The calculational techniques are described in Sec. III and the results of numerical simulations for the bilayer model are presented in Sec. IV. Finally, the results are discussed in Sec. V, which contains a summary of extensions to lipid-protein interactions and concludes the paper.

\section{MICROSCOPIC MODEL}

The Hamiltonian for the model used in this paper describes both monolayers of a bilayer and allows for interactions between the monolayers. The principal term in the Hamiltonian describing a single monolayer is the tenstate lattice model due to Pink $[6,7]$. The Pink model takes into account the acyl-chain conformational statistics and the van der Waals interactions between various conformers in a detailed manner, while the excludedvolume effect is partially accounted for by assigning each lipid chain to a single site of a triangular lattice. The acyl-chain conformations are represented by ten single- 
chain states, $\alpha=1, \ldots, 10$, each described by a crosssectional area $A_{\alpha}$, an internal energy $E_{\alpha}$, and an internal degeneracy $D_{\alpha}$. The nine lower states are characteristic of the gel phase and the tenth state is typical of the fluid phase. Of the nine gel states, the lowest state is the all-trans ground state and the eight remaining states are low-lying conformational excitations of the ground state. The Hamiltonian for two noninteracting monolayers can be written as follows:

$$
\begin{aligned}
\mathcal{H}_{o}=\sum_{n=1}^{2} & \left(\sum_{i} \sum_{\alpha=1}^{10}\left(E_{\alpha}+\Pi A_{\alpha}\right) \mathcal{L}_{\alpha, i}^{n}\right. \\
& \left.-\frac{J_{0}}{2} \sum_{\langle i, j\rangle} \sum_{\alpha, \beta=1}^{10} I_{\alpha} I_{\beta} \mathcal{L}_{\alpha, i}^{n} \mathcal{L}_{\beta, j}^{n}\right),
\end{aligned}
$$

where $\langle i, j\rangle$ are nearest-neighbor indices. $J_{0}$ is the strength of the van der Waals interaction. $\mathcal{L}_{\alpha, i}^{n}$ is an occupation variable which is unity when the $i$ th chain of the $n$th monolayer is in the $\alpha$ th conformational state and zero otherwise. $I_{\alpha}$ is a product of a term related to the van der Waals interaction between chains and a phenomenological expression for the shape-dependent nematic order parameter due to Seelig [19]. II represents the effective lateral pressure exerted on a lipid bilayer due to interfacial effects (hydration, polar head interactions, etc.). The parameters $J_{0}$ and $\Pi$ were found by fitting to experimental values for the transition temperatures and transition enthalpies of pure saturated PC bilayers. The values found for dipalmitoyl phospatidylcholine are $J_{0} \approx 0.71 \times 10^{-13} \mathrm{erg}$ and $\Pi=30 \mathrm{dyn} / \mathrm{cm} \mathrm{[5].} \mathrm{It} \mathrm{turns}$ out that with these parameter values the model is very close to a critical point on the side where there is no transition. This is consistent with the interpretation of the most accurate thermodynamic data available [15].

The Hamiltonian $\mathcal{H}_{m}$, which describes the mismatch interaction between different conformational states of the acyl chains across the bilayer and hence accounts for intermonolayer coupling in an indirect way, is assumed, by analogy with the case of lipid-protein interactions [14], to have the following form:

$$
\mathcal{H}_{m}=\frac{\gamma_{\text {mis }}}{2} \sum_{\langle i, j\rangle} \sum_{\alpha 1, \beta 1} \sum_{\alpha 2, \beta 2}\left|d_{\alpha 1}+d_{\beta 1}-d_{\alpha 2}-d_{\beta 2}\right| \mathcal{L}_{\alpha 1, i}^{1} \mathcal{L}_{\beta 1, i}^{2} \mathcal{L}_{\alpha 2, j}^{1} \mathcal{L}_{\beta 2, j}^{2}
$$

where $d_{\alpha}$ is the hydrocarbon chain length for the $\alpha$ th conformational state, which is reciprocally related to the value of $A_{\alpha}$ since the volume of an acyl chain is assumed invariant under temperature changes. The parameter $\gamma_{\text {mis }}$ is related to the hydrophobic effect.

Finally, we consider a Hamiltonian $\mathcal{H}_{\mathrm{i}}$ which represents a direct contribution from the interlayer coupling. We assume that every acyl chain interacts with its nearest neighbors in the opposite monolayer in a pairwise manner with an energy $-J_{2} \Omega_{\alpha} \Omega_{\beta}$, where $\Omega_{\alpha}$ are statedependent parameters. In the fluid phase the acyl chains are disordered and the lateral diffusion coefficient is at least two orders of magnitude greater than in the gel phase [17]. This implies that the interlayer coupling between the chains in the gel state is much greater than that in fluid state. We therefore take $\Omega_{1}=1, \Omega_{10}=0$ and chose $\Omega_{2,3,4}=\frac{2}{3}, \Omega_{5,6,7}=\frac{1}{3}$, and $\Omega_{8,9}=\frac{1}{9} \cdot \mathcal{H}_{i}$ can be written as follows:

$$
\mathcal{H}_{i}=J_{2} \sum_{i} \sum_{\alpha, \beta} \Omega_{\alpha} \Omega_{\beta} \mathcal{L}_{\alpha, i}^{1} \mathcal{L}_{\beta, i}^{2}
$$

The total Hamiltonian of the model used here is therefore

$$
\mathcal{H}=\mathcal{H}_{o}+\mathcal{H}_{m}+\mathcal{H}_{i}
$$

The particular system considered here is a single DPPC bilayer. With appropriate scaling according to acyl-chain length, the results presented should be applicable to other phosphatidylcholine bilayers [8]. The values used for the coupling constants of the mismatch interaction and the interlayer coupling are given below.

\section{CALCULATIONAL TECHNIQUES AND PHYSICAL QUANTITIES}

In this section we describe the numerical techniques used to identify the nature of the transition and the phys- ical quantities calculated in the neighborhood of the transition. We begin with an analysis of first-order transitions which are characterized by discontinuities in the first derivatives of the free energy. Specifically, a $\delta$-function singularity occurs in specific heat $C(T)$ at the transition in the thermodynamics limit. In a finite system, however, the transition region is broadened and the peak in $C(T)$ as well as in the isothermal compressibility $\chi(T)$ has a finite intensity which increases with increasing linear lattice size $L$. Furthermore, the location of the maximum varies in a size-dependent manner. The maximum grows as $L^{d}$ in $d$ dimensions and the $\delta$-function limit is obtained because the width decreases simultaneously as $L^{-d}$. The maximum value of $C(T)$ for a finite lattice is

$$
C_{L}^{\max }=a+b L^{d}
$$

where $a$ and $b$ are the size-independent parameters characteristic of the specific system [20]. A similar scaling form holds for the maximum value of the isothermal compressibility $\chi_{L}^{\max } . C(T)$ and $\chi(T)$ are calculated from the fluctuation-dissipation theorem

$$
\begin{aligned}
& \chi(T)=\frac{1}{k_{B} T}\left(\left\langle A^{2}\right\rangle-\langle A\rangle^{2}\right) / L^{d}, \\
& C(T)=\frac{1}{k_{B} T^{2}}\left(\left\langle\mathcal{H}^{2}\right\rangle-\langle\mathcal{H}\rangle^{2}\right) / L^{d},
\end{aligned}
$$

where

$$
A=\sum_{\alpha, i} A_{\alpha} \mathcal{L}_{\alpha, i}
$$

The phase behavior of the Pink model of Eq. (1) was previously examined by the standard Metropolis Monte Carlo method with importance sampling and Glauber dy- 
namics [5]. This method is used here in conjunction with the Monte Carlo extrapolation method of Ferrenberg and Swendsen [21] and the finite-size scaling method due to Lee and Kosterlitz $[10,11]$ to examine the nature of the phase transition for the model in Eq. (4). The Lee and Kosterlitz method consists of calculating the free energy as a function of the order parameter at the transition using the probability distribution of the order parameter at the temperature where the specific heat peak occurs. For the present model we use the average area $\langle A\rangle$ per lipid chain as the order parameter since $\langle A\rangle$ is coupled to the internal lateral pressure $\Pi$ [see Eq. (1)] $[9,12]$. At a first-order transition, the bulk free energy $\mathcal{F}(A, L)$ has pronounced double minima corresponding to two coexisting phases at $A=A_{1,2}$ separated by a maximum at $A_{\max }$ corresponding to a domain wall between the two phases. The maximum height is

$$
\Delta \mathcal{F}(L)=\mathcal{F}\left(A_{\max }, L\right)-\mathcal{F}\left(A_{1}, L\right) \sim L^{d-1} .
$$

At a first-order transition, $\Delta \mathcal{F}(L)$ increases monotonically with $L[12]$.

The above method was used in our earlier work [12] to examine the phase behavior of an extended Pink model for a single monolayer with intramonolayer mismatch interactions. Here we apply this method to the full bilayer model and furthermore examine the state of the bilayer in detail near the transition. For this purpose we investigate cluster-formation phenomena [5] and calculate the chain orientational order parameter in the neighborhood of the transition. The clusters can be described by a size-distribution function $n_{\ell}^{p}(T)$ which gives the number of clusters of type $p$ with $\ell$ lipid chains. Here $p$ refers to the fluid chain state if $T<T_{m}$ or the gel and intermediate chain states if $T>T_{m}$ [5]. The clusters are defined via a nearest-neighbor connectivity criterion dictated by the interaction range. The average cluster size is then

$$
\xi(T)=\sum_{\ell} \ell n_{\ell}^{p}(T) / \sum_{\ell} n_{\ell}^{p}(T),
$$

where the summation is restricted by a lower cutoff in the value of $\ell$ (corresponding to three acyl chains in our case) in order to exclude local fluctuations controlled by the Glauber dynamics. We analyze the spatial pattern of the lipid clusters by dividing each monolayer into three regions [5]: the background phase (bulk $b$ ), the clusters $(c)$, and the interface $(i)$ between the clusters and the bulk. The interfacial region is composed of those acyl chains which have nearest-neighbor bonds to the cluster boundary. The spatial pattern is then described in terms of the corresponding fractional areas of the membrane, $a_{b}, a_{c}$, and $a_{i}$, and the relative occurrences of acyl-chain states in the different regions.

The chain orientational order parameter $S$ is calculated as follows:

$$
S=L^{-2} \sum_{n=1}^{2} \sum_{i} \sum_{\alpha=1}^{10}\left(\omega_{1} A_{1} A_{\alpha}^{-1}+\omega_{2}\right) \mathcal{L}_{\alpha, i}^{n},
$$

where $\omega_{1}=1.8$ and $\omega_{2}=-0.8$ [5]. Equation (11) is derived on the basis of geometrical considerations involv- ing the embedding of the acyl chains on a triangular lattice [19]. In order to obtain values of $S$ for a large system over a wide temperature range, we computed this quantity by using the standard Monte Carlo method without using the Ferrenberg-Swendsen method.

\section{RESULTS}

In this section we present simulation results for the model of a bilayer consisting of two interacting monolayers as described by the Hamiltonian of Eqs. (1) to (4). The results are derived from simulations using several different lattice sizes, $L \times L$, subject to periodic boundary conditions. Very long simulations were performed at the transition temperature in order to obtain good statistics $[10,20]$. The temperatures at which the simulations are performed were chosen as close as possible to the transition temperature $T_{m}(L)$. It was found that $2 \times 10^{6}$ Monte Carlo steps per site (MCS) were sufficient to obtain the required accuracy.

The temperature at which a finite system undergoes a first-order phase transition usually depends on the size of the system. This transition temperature $T_{m}(L)$ is defined by requiring $\mathcal{F}\left(A_{1}, L\right)=\mathcal{F}\left(A_{2}, L\right)$, where $\mathcal{F}\left(A_{1}, L\right)$ and $\mathcal{F}\left(A_{2}, L\right)$ are the free energies of the gel and fluid phases, respectively. The free energies are first calculated for varying values of the mismatch parameter $\gamma_{\text {mis }}$ and for a fixed value of the interlayer coupling $J_{2}$ in order to examine the phase behavior of the system and to find the critical point. A value of $J_{2}=0.04 J_{0}$ was chosen in accordance with earlier work on intermonolayer coupling [17]. We found that the system did not exhibit a phase transition for values of $\gamma_{\text {mis }}$ below $3 \times 10^{-16} \mathrm{erg} / \AA$ for $J_{2}=0.04 J_{0}$. In the subsequent calculations, we then chose a value of $4 \times 10^{-16} \mathrm{erg} / \AA$ for $\gamma_{\text {mis }}$ at which the system exhibits a weak first-order phase transition. This is illustrated in Fig. 1 which gives the free energy as a function of area per lipid chain for these parameters in the case of several lattice sizes varying from $L=8$ to 24 .

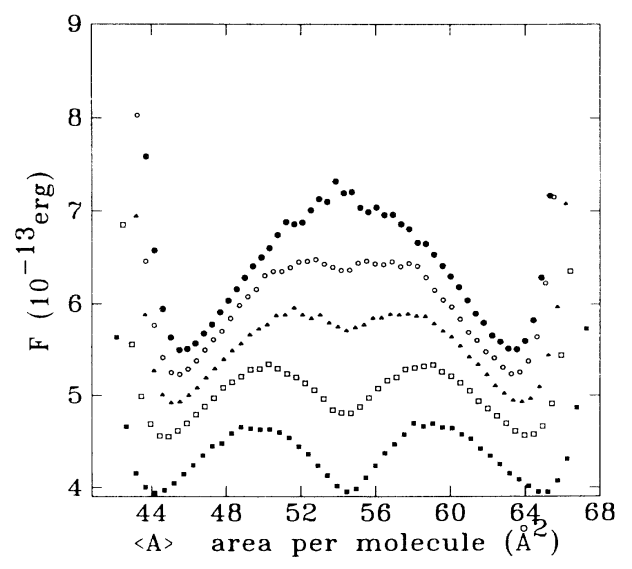

FIG. 1. Free energy $\mathcal{F}\left(T_{\mathrm{m}}(L)\right)$ as a function of order parameter $\langle A\rangle$ for lattice sizes $L \times L, L=8,12,16,20,24$ from bottom to top, for $\gamma_{\mathrm{mis}}=4 \times 10^{-16} \mathrm{erg} / \AA$, and $J_{2} / J_{0}=0.04$. $T_{m}(L)$ refers to the transition temperature for a system of linear size $L$. 


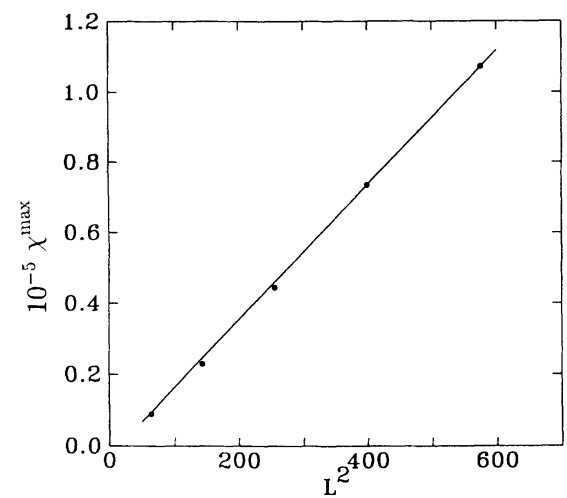

FIG. 2. Variation of the specific-heat maximum with $L^{2}$ for $L=8,12,16,20,24$.

This figure shows that there is a finite-size effect for the smaller lattice sizes, i.e., an additional well is present because the monolayers are in different phases, one being in a gel phase and the other in a fluid phase. Hence in this case, the weak coupling between the finite-size monolayers is overcome by strong lateral fluctuations within the monolayers. Figure 1 also shows that the additional well disappears as the size of system increases, since the interlayer coupling then ensures that the entire bilayer is in either the gel phase or in the fluid phase at any given temperature. It can be seen from the figure that the two phases are separated by an energy barrier whose height increases with increasing $L$. This indicates that the system undergoes a first-order phase transition in the thermodynamic limit [10]. The variation of the specific heat and the susceptibility maxima with $L$ are shown for several lattice sizes in Figs. 2 and 3, respectively. These figures show that both response functions are linear in $L^{2}$, as expected for a first-order transition (see [20] and Eq. (5)).

In the rest of this section we describe the effect of interlayer interactions on the dynamic heterogeneity of the bilayer. For this purpose we perform a series of calculations in the neighborhood of the specific-heat peak using the Ferrenberg-Swendsen extrapolation method (see [21]

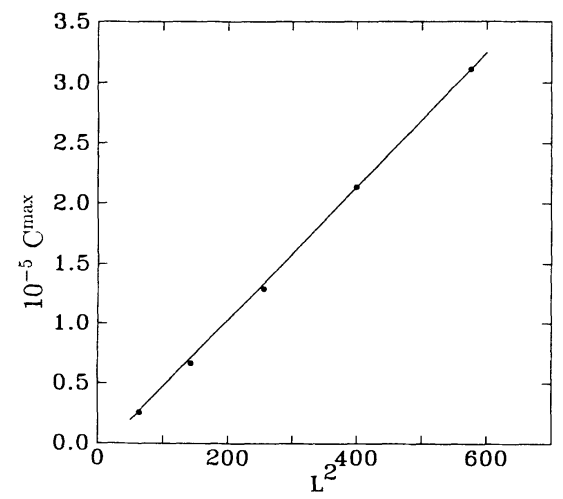

FIG. 3. Variation of the susceptibility maximum with $L^{2}$ for $L=8,12,16,20,24$.

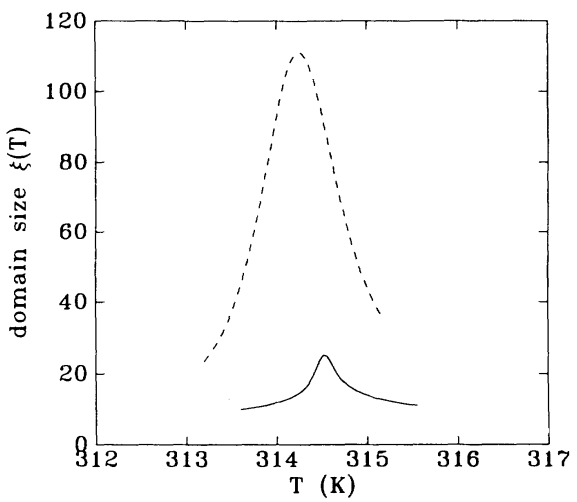

FIG. 4. Average domain size (number of chains), $\xi(T)$ in Eq. (10), as a function of temperature for the Pink model (dashed line) and the model with intermonolayer interactions (solid line), respectively. The curves are obtained from Ferrenberg-Swendsen simulations on a $24 \times 24$ lattice. The lower cutoff cluster size is taken to be three acyl chains.

and Sec. III) for a $24 \times 24$ lattice both in the presence and absence of these additional interactions. Note that the absence of the additional interactions corresponds to the case of an isolated DPPC monolayer which for our parameters does not exhibit a phase transition in the thermodynamic limit, but remains close to the critical point. Figure 4 shows the average linear dimension, $\xi$ in Eq. (10), of the fluctuating clusters of the minority phase as a function of temperature for both cases. It is seen that $\xi$ is considerably reduced at any given temperature in the presence of the additional interactions, implying that the transition region has been considerably narrowed and that the "wings" of the transition are confined to a much narrower temperature range. This is understandable because of the high energy barrier for the formation of cluster interfaces in a first-order transition.

Figure 5 shows that the fraction of the membrane area in the clusters and in the interfaces is considerably lower

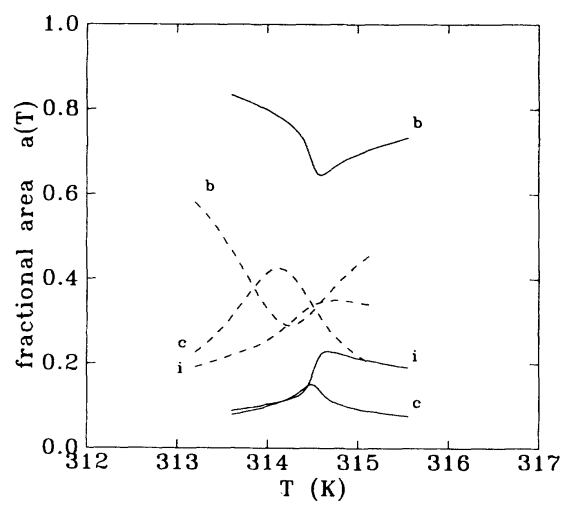

FIG. 5. Temperature dependence of the fractional areas $a(T)$ in the bulk $(b)$, in the clusters $(c)$, and in the first interfacial layer $(i)$ for the Pink model (dashed lines) and for the model with interlayer interactions (solid lines). Both sets of results are obtained from a $24 \times 24$ lattice using the Ferrenberg-Swendsen method. 


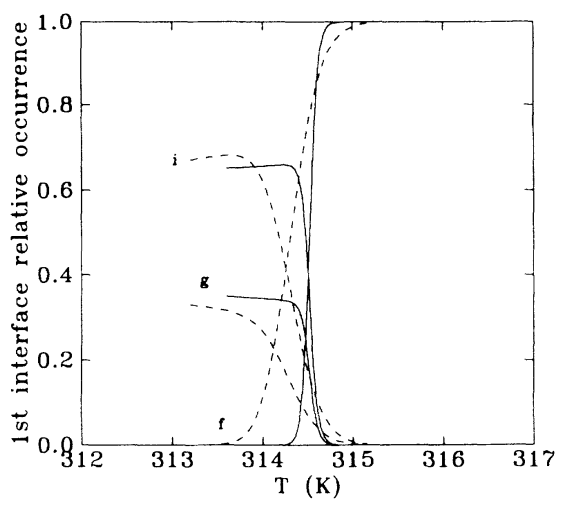

FIG. 6. Relative occurrence of gel $(g)$, intermediate $(i)$, and fluid $(f)$ acyl-chain conformational states in the first interfacial layer of lipids chains for the Pink model (dashed lines) and for the model with interlayer interactions (solid lines). The results are obtained from a $24 \times 24$ lattice using the Ferrenberg-Swendsen method.

in the case of a first-order phase transition induced by the intermonolayer interactions. This figure also shows that the fractional areas of the bulk, interface, and cluster regions are similar close to $T_{m}$ in the absence of a phase transition, whereas in the case of a first-order transition the bulk dominates in this temperature range. The interfacial regions can be probed by calculating the relative occurrence of the acyl-chain conformational states in the first interfacial layer between the clusters and the bulk. This layer is defined as the set of acyl chains which are connected by nearest-neighbor lattice bonds to the cluster boundary. Figure 6 shows the relative occurrence of the all-trans, the intermediate, and the fluid states in the first interfacial layer. The same quantities are given for the bulk in Fig. 7. A comparison of Figs. 6 and 7 demonstrates that the first interfacial layer is dominated by chains in intermediate conformational states. A soft interface of this kind in a biological lipid bilayer could

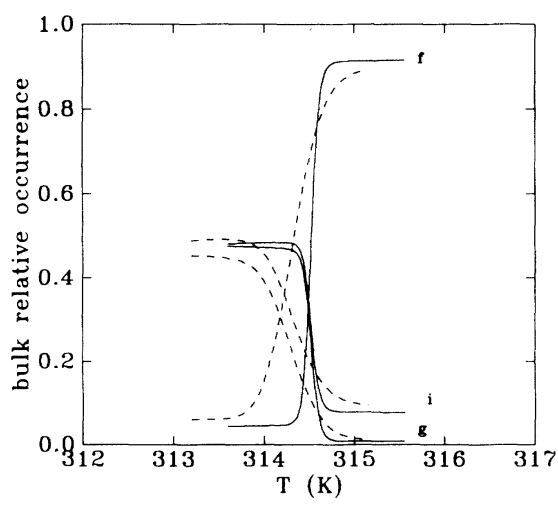

FIG. 7. Relative occurrence of gel $(g)$, intermediate $(i)$, and fluid $(f)$ acyl-chain conformational states in the bulk for the Pink model (dashed lines) and for the model with interlayer interactions (solid lines). The results are obtained from a $24 \times 24$ lattice using the Ferrenberg-Swendsen method.

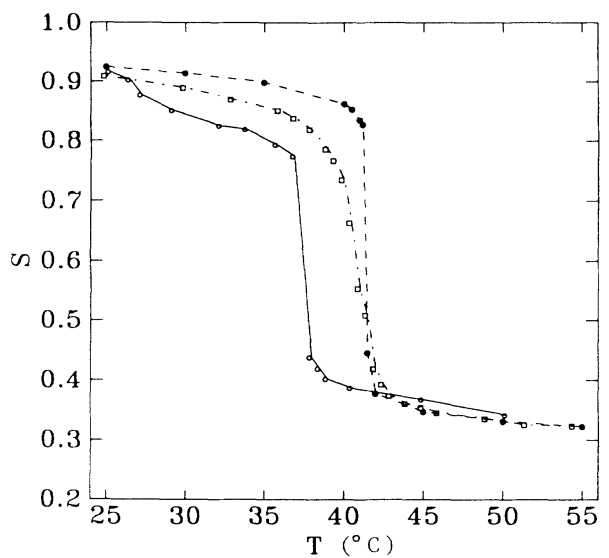

FIG. 8. Acyl-chain orientational order parameter $S$ as a function of temperature. Theoretical results, Eq. (11), are shown as obtained from model simulations on a $60 \times 60$ bilayer for the Pink model $(\square)$ and for the model with interlayer interactions $(\bullet)$. Experimental data $(\circ)$ are shown for comparison as obtained from the first moment of the quadrupolar nuclear magnetic resonance spectrum of $d_{62}$-DPPC lipid bilayers (Ref. [22]).

very well have some biological significance [5]. These figures also show that the variation with temperature in the neighborhood of $T_{m}$ of the relative occurrences of the different chain states are similar in the absence and in the presence of a first-order phase transition, although considerably sharper in the latter case.

We have checked the validity of the application of the Ferrenberg-Swendsen method to examine the bilayer heterogeneity near $T_{m}$ by performing standard Metropolis Monte Carlo simulations for the quantities shown in Figs. 5-7 in the case of a first-order phase transition. The results obtained show that there is good agreement between the two methods except at $T_{m}$. This minor disagreement is due to hysteresis effects which are present near the first-order phase transition in our standard Monte Carlo calculations due to limited statistics.

In Fig. 8 we present the results of the acyl-chain orientational order parameter $S$, which is calculated from Eq. (11) both in the absence and presence of a first-order phase transition. We also show the experimental data of Davis [22] for $S$ obtained from the first moment of the quadrupolar nuclear magnetic resonance spectrum of $d_{62^{-}}$ DPPC bilayers. The experimental order-parameter data exhibit a sharp variation in the transition region. The model results for $S$ found by including interlayer interactions also exhibit a sharp jump at $T_{m}$, which, however, is in closer agreement with the experimental results. In contrast, the single monolayer model gives a curve for $S$ which is much smoother than the experimental results. The reason for the difference in the value of $T_{m}$ between the experimental and theoretical results is trivially related to the fact that the experiments were performed on fully deuterated chains whereas the theory applies to fully protonated chains for which $T_{m}$ is higher. The difference in the theoretical and experimental variation of $S$ with temperatures below $T_{m}$ is due to the fact that the 
pretransition of DPPC bilayers has not been considered in the theoretical analysis, whereas it clearly affects the experimental results.

\section{CONCLUSION}

We have proposed and examined a bilayer model composed of two monolayers each of which is modeled by the ten-state Pink Hamiltonian of Eq. (1). We also included interlayer interactions between the two monolayers, Eqs. (2) and (3). The intermonolayer interactions included both a mismatch term which assured compatibility of the monolayers as well as a term which coupled directly the two monolayers. The strength of the intermonolayer interaction was chosen in such a way that the bilayer would not have a phase transition in the thermodynamic limit in the absence of interlayer coupling but was extremely close to a critical endpoint [12]. In order to observe a first-order phase transition, a nonzero value of the interlayer coupling constant is required. Both parts of the intermonolayer coupling tends to drive the transition first order but the mismatch interaction is the more effective one. The Ferrenberg-Swendsen method was then used to examine the bilayer heterogeneity near the phasetransition temperature. The results were compared with the case where the interlayer coupling are absent. In this case there is no phase transition in the thermodynamic limit and the critical fluctuations are present in the form of fluctuating clusters of the minority phase in the majority phase, leading to a considerable dynamic heterogeneity. The first-order phase transition induced by the interlayer coupling results in a more homogeneous bilayer with a much narrower transition region, a considerable reduction of the "wings" of the transition, and much reduced fluctuating clusters at any given temperature. Furthermore, the orientational order parameter $S$ has in the case of the first-order transition a very abrupt decrease at $T_{m}$ in agreement with experiment. A direct experimental determination of the pseudocritical fluctuations in lipid bilayers close to $T_{m}$ would therefore be of great help in probing the nature of the main phase transition.

The bilayer model presented in this paper should be of use in further studies of lipid membranes where the transmonolayer coupling is of crucial importance. Examples include the phase behavior of asymmetric bilayers, as well as the behavior of transmembrane integral proteins, polypeptides, and ion channels.

\section{ACKNOWLEDGMENTS}

This work was supported by FCAR du Quebec under a centre and team grant, by NSERC of Canada, and by the Danish Natural Science Research Council under Grant No. 11-7785. The Danish Research Academy supported M.J.Z. and Z.Z. during their stay in Denmark under Grant No. S910015.
[1] G. Cevc and D. Marsh, Phospholipid Bilayers. Physical Principles and Models (Wiley-Interscience, New York, 1987).

[2] M. Bloom, E. Evans, and O. G. Mouritsen, Q. Rev. Biophys. 24, 293 (1991).

[3] O. G. Mouritsen, Chem. Phys. Lipids 57, 158 (1991).

[4] E. Egberts and H. J. C. Berendsen, J. Chem. Phys. 89, 3718 (1988).

[5] O. G. Mouritsen, in Molecular Description of Biological Membrane Components by Computer Aided Analysis, edited by R. Brasseur (CRC Press, Boca Raton, FL, 1990), Vol. 1, pp. 3-83.

[6] D. A. Pink, T. J. Green, and D. Chapman, Biochem. 20, 6692 (1981).

[7] A. Caillé, D. A. Pink, F. de Verteuil, and M. J. Zuckermann, Can. J. Phys. 58, 581 (1980).

[8] J. H. Ipsen, K. Jørgensen, and O. G. Mouritsen, Biophys. J. 58, 1099 (1990).

[9] E. Corvera, M. Laradji, and M. J. Zuckermann (unpublished).

[10] J. Lee and J. M. Kosterlitz, Phys. Rev. Lett. 65, 137
(1990).

[11] J. Lee and J. M. Kosterlitz, Phys. Rev. B 43, 3265 (1991).

[12] Z. Zhang, M. Laradji, H. Guo, O. G. Mouritsen, and M. J. Zuckermann, Phys. Rev. A 45, 7560 (1992).

[13] O. G. Mouritsen and M. Bloom, Biophys. J. 46, 141 (1984).

[14] M. M. Sperotto and O. G. Mouritsen, Eur. Biophys. J. 19, 157 (1991).

[15] R. L. Biltonen, J. Chem. Thermodyn. 22, 1 (1990).

[16] M. R. Morrow, J. P. Whitehead, and D. Lu, Biophys. J. 63, 18 (1992).

[17] A. Georgallas, D. L. Hunter, T. Lookman, M. J. Zuckermann, and D. A. Pink, Eur. Biophys. J. 11, 79 (1984).

[18] S. Doniach, J. Chem. Phys. 68, 4912 (1978).

[19] J. Seelig and A. Seelig, Q. Rev. Biophys. 13, 19 (1980).

[20] M. S. S. Challa, D. P. Landau, and K. Binder, Phys. Rev. B 34, 1841 (1986).

[21] A. M. Ferrenberg and R. H. Swendsen, Phys. Rev. Lett. 61, 2635 (1988).

[22] J. H. Davis, Biophys. J. 27, 339 (1979). 